\title{
Multisystem Inflammatory Syndrome in an Adult During the COVID-19 Pandemic
}

Ritu Chakrabarti, MD' - Manlio Hernandez Castillo, MD' - Aaisha Shah, MD' - Marissa Sansone, MD² - Valentin Marian, MD³

\begin{abstract}
A 27-year-old woman with no significant medical history presented with sudden-onset midsternal chest pain radiating to her arms bilaterally, dizziness, diffuse band-like headache, intermittent blurry vision, and shortness of breath. She reported a 1-week history of a dry, nonproductive cough and cold sweats.

The patient had presented to our clinic during the peak of the COVID-19 pandemic and had frequently used public transportation in New York City. Her family history was nonsignificant for autoimmune conditions or other diseases.
\end{abstract}

\section{Physical examination}

Upon admission to the hospital, she had hypotension with a low-grade fever and dyspnea with bilateral rales. Laboratory findings were significant for leukocytosis, lactic acidosis, and an elevated troponin I level. Her d-dimer level was within the normal range, and test results were negative for antinuclear antibody, smooth muscle antibody, and immunoglobulin G4 (IgG-4). Results of a COVID-19 polymerase chain reaction (PCR) test were also negative.
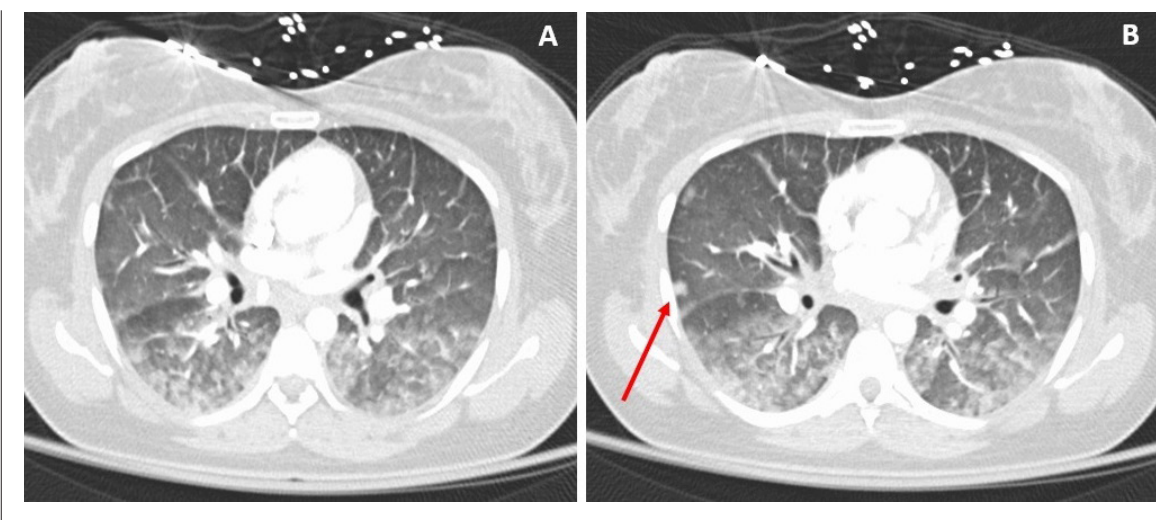

Figure 1. A computed tomography scan showed extensive bilateral, posterior lung opacities with infiltrate process involving the mediastinum, suggestive of pneumonia (A). A 7-mm nodule was also noted in periphery of the right middle lobe (B).

\section{Diagnostic testing}

The patient's initial electrocardiography (EKG) scan showed ST depressions in leads II, III, AVF, and V3 to V6. She was emergently treated with ticagrelor, 180 $\mathrm{mg}$; heparin, $5000 \mathrm{U}$; and aspirin, $324 \mathrm{mg}$, in preparation for potential percutaneous coronary intervention. A diagnostic cardiac catheterization subsequently showed normal coronary arteries but moderately reduced ejection fraction (45\%) with some wall motion abnormalities.

\section{AFFILIATIONS:}

'Department of Internal Medicine, Jersey City Medical Center, Jersey City, New Jersey ${ }^{2}$ Department of Rheumatology, Yale University School of Medicine, New Haven, Connecticut ${ }^{3}$ Department of Rheumatology, Jersey City Medical Center, Jersey City, New Jersey

\section{CITATION:}

Chakrabarti R, Hernandez Castillo M, Shah A, Sansone M, Marian V. Multisystem inflammatory syndrome in an adult during the COVID-19 pandemic. Consultant. Published online August 25, 2021. doi:10.25270/ con.2021.08.00010

Received March 30, 2021. Accepted April 26, 2021.

\section{DISCLOSURES:}

The authors report no relevant financial relationships.

\section{CORRESPONDENCE:}

Ritu Chakrabarti, MD, Jersey City Medical Center, 355 Grand Street, Jersey City, NJ 07302 (Ritu.chakrabarti@rwjbh.org)
Results of an echocardiography scan confirmed global hypokinesis, indicative of myocarditis. The patient's hypotension and tachypnea worsened. A computed tomography angiogram of the chest did not show a pulmonary embolism but was significant for extensive bilateral, posterior lung opacities with infiltrative processes involving the mediastinum, suggestive of possible pneumonia or mediastinitis with bilateral lung nodules (Figure 1).

The thymic gland and pancreas were also prominent on the scans, suggestive of thymic hyperplasia and autoimmune pancreatitis, respectively, hence our rationale for evaluating her IgG-4 levels (Figure 2). During this time, the patient began displaying new vertical nystagmus. A magnetic resonance imaging (MRI) scan of the brain illustrated small areas of abnormal signaling in the right frontal lobe (Figure 3). The patient was transferred to the critical care unit for further management and was stabilized with intravenous fluids and antibiotics including azithromycin, $500 \mathrm{mg}$, daily and ceftriaxone, $1 \mathrm{mg}$, daily for 5 days.

Given the complexity of the case, a 

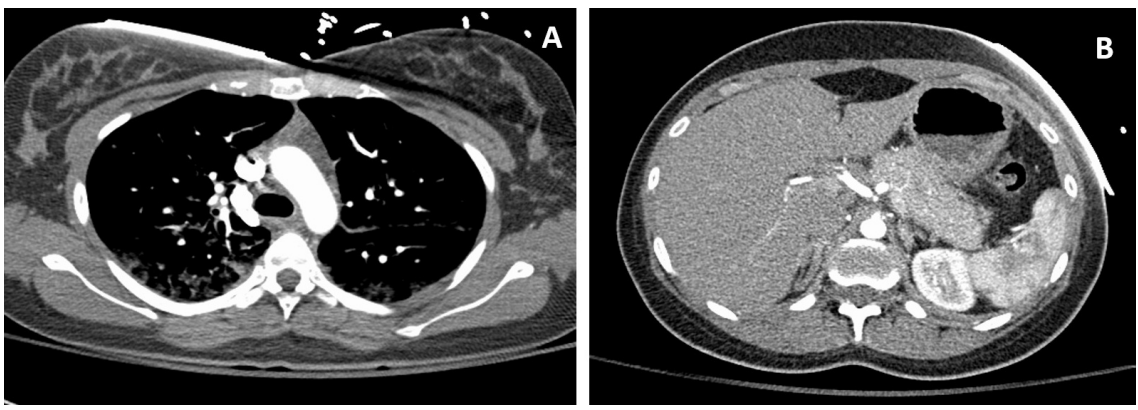

Figure 2. A computed tomography scan showed prominence of the thymic gland, presumably because of thymic hyperplasia (A). Prominence of the pancreas was also noted, with loss of interdigitations (B).

neurologist and rheumatologist were consulted. At the time, the differential diagnoses included a post-viral inflammatory syndrome or a systemic autoimmune process. Further laboratory tests were conducted-including tests for Coxsackie $A$ and $B$ and mycoplasma, urine antigen for legionella and pneumococcus species, rapid antigen reagin titers, Lyme titers, and cultures (cerebrospinal fluid, sputum, blood)-but all results were negative or noncontributory. A repeat COVID-19 nasopharyngeal PCR test was conducted, as well as a SARS-CoV-2 $\lg G$ antibody test, results of which were both negative.

Because no infectious etiology was found, our patient was presumed to have a systemic inflammatory syndrome of unknown etiology and thus was started on high-dose prednisone and transitioned to complete a 5-day course of oral azithromycin and ceftriaxone. Our patient started showing improvement in clinical signs and symptoms, as well as laboratory findings, over the next 3 days and was discharged home on prednisone, $40 \mathrm{mg}$, daily with tapering doses over the following 2 weeks.

\section{Discussion}

As the COVID-19 pandemic continues to affect global populations, we are uncovering different clinical presentations and disease sequelae associated with SARS-CoV-2 infection. While the virus typically presents with a severe acute respiratory syndrome, it has been shown to have tropism for different organ systems, including the lungs, small intestines, and kidneys, as well as cause systemic inflammation. Some children who had developed a Kawasaki-like illness with fever, abdominal pain, and conjunctivitis along with multiorgan involvement and laboratory findings significant for inflammation were later diagnosed with COVID-19 based on serology!' Initially reported from Europe in children and adolescents, this hyperinflammatory syndrome has been characterized as multisystem inflammatory syndrome in children (MIS-C) by the Centers for Disease Control and Prevention.' Interestingly, cases of the same entity have been reported in adults. ${ }^{2}$

In December 2019, SARS-CoV-2, part of the Coronaviridae family of positive-sense single-stranded RNA viruses, was identified to be the cause of severe pneumonia in a cluster of patients in Wuhan, China. It then spread globally and was declared a pandemic by the World Health Organization in March 2020. ${ }^{3}$ The virus triggers an array of symptoms with multiorgan involvement, including pneumonitis and acute respiratory distress syndrome, myocarditis and heart failure, neurological manifestations, renal failure, and gastrointestinal involvement. ${ }^{4-10}$ COVID-19 evades the body's natural defense mechanisms by causing a delayed and downregulated interferon-stimulated gene expression, thereby dampening the body's cellular defense mechanisms and the innate immune system's early response against pathogens." Subsequently, robust cytokine release creates a proinflammatory state that causes multiorgan damage and rapid clinical deterio- ration with high mortality rates.2,13 Many immune-mediated disorders have been associated with COVID-19, including a multisystem inflammatory Kawasaki-like syndrome first identified in children admitted to a hospital in northern Italy. ${ }^{14}$ This has since been defined as MIS-C and is characterized by the following:

- Age younger than age 21 years with presenting symptoms of fever, evidence of severe illness requiring hospitalization, multisystem organ involvement (2 or more of the following: cardiac, renal, respiratory, hematologic, gastrointestinal, dermatologic, or neurologic)

- No alternative diagnosis

- A positive COVID-19 test by reverse transcription PCR, serology, or antigen tests or exposure to a suspected or confirmed case of COVID-19 within 4 weeks prior to onset of symptoms

Herein, we present a case of a 27-yearold woman with a post-viral immunemediated inflammatory process characterized by pneumonitis, myocarditis, encephalitis, and subclinical pancreatitis that was responsive to steroids, closely resembling MIS-C. While our patient's COVID-19 PCR test result was negative, it is important to note her high likelihood of exposure to the virus, since she was using New York City public transportation daily during the peak of the COVID-19 pandemic. The viral prodrome for 1-week, bilateral patchy opacities seen on imaging and initial hypotension make a further compelling case for initial asymptomatic COVID-19 infection followed by MIS in an adult, despite initial negative PCR or IgG laboratory test results. There have been well-documented cases of patients fulfilling criteria for MIS-C without confirmed COVID-19 PCR or IgG findings. ${ }^{15,16}$

The cardiac findings in our patient-including the initial EKG changes suggestive of ischemia, elevated troponin and NT-pro-BNP levels, and global hypokinesis with reduced ejection fraction-and subsequent resolution of wall motion 

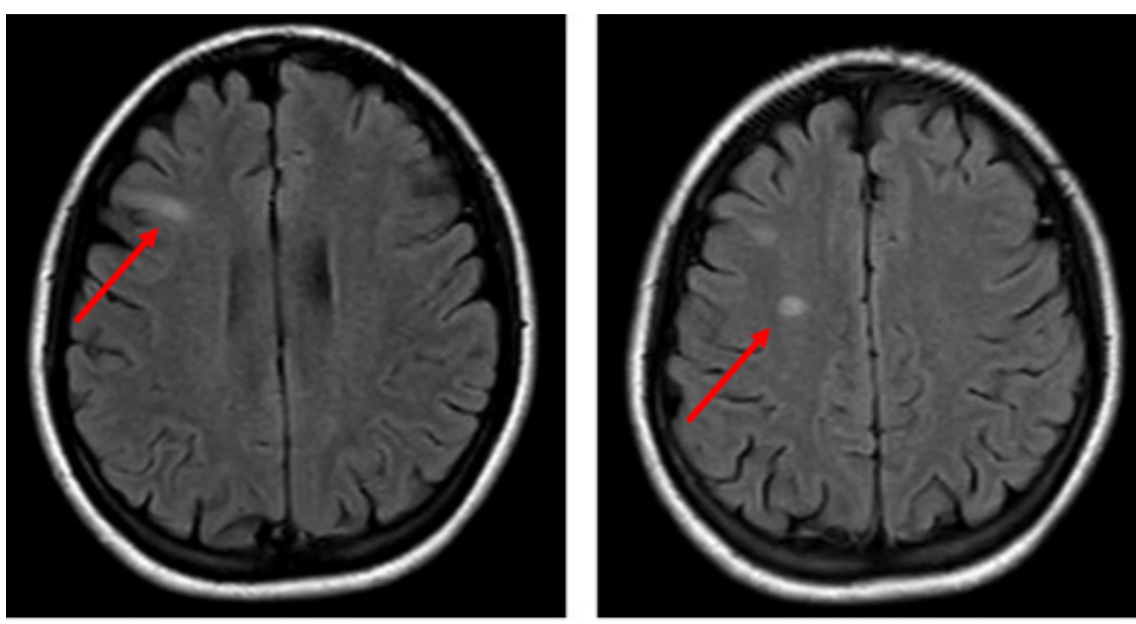

Figure 3. A magnetic resonance imaging scan showed small areas of abnormal signaling in the right frontal lobe.

abnormalities are diagnostic for acute myocarditis and has been well-documented in children with MIS-C. ${ }^{17}$ Our patient's multifocal neurological deficits of vertical nystagmus, diplopia, and MRI hyperintensities are suggestive of immune-mediated central nervous system inflammation like that seen in acute disseminated encephalomyelitis, which may have been ameliorated by early steroid use. Similar episodes of encephalopathy, cerebellar signs, and even cerebral infarct have been documented in MIS-C cases with negative cerebrospinal fluid findings. ${ }^{15,18,9}$ Our patient's C-reactive protein level was elevated upon admission, but her erythrocyte sedimentation rate was within normal limits, which may have suggested a very early inflammatory process. Her complete resolution of symptoms in the setting of steroid administration also suggests a monophasic inflammatory syndrome, possibly post-infectious, vs autoimmune rheumatic syndromes that may relapse over time.

Interestingly, a new case series described 27 patients who fulfilled criteria for what the researchers defined as MIS-A: multisystem inflammatory syndrome in adults. ${ }^{2}$ Of note, many of these patients did not have respiratory symptoms of COVID-19, had extrapulmonary organ dysfunction, high inflammatory markers, and required corticosteroids much like our patient. COVID-19 antibody testing returned positive results in many of these patients, demonstrating a possible post-infectious or alternative organ involvement of the virus.

As we learn more about the effects of COVID-19 on the immune system and multiple organ systems, it will be essential to maintain high clinical suspicion for a post-viral inflammatory syndrome, as well as to continue to elucidate further diagnostic methods. Brain MRI scanning has been shown to display characteristic findings in patients with COVID-19 who demonstrate neurologic symptoms without cerebral ischemia. ${ }^{20,21}$ Further, cardiac MRI scanning has been shown to be a useful surveillance methodology for patients with heart involvement. ${ }^{22}$

\section{Patient outcome}

Interestingly, our patient's symptoms completely resolved when she followed up with the rheumatologist in the outpatient setting 1 week after discharge. However, one day after completing the tapered steroid regimen, the patient returned to the emergency department with chest pain and vertigo with nystagmus similar to her previous presentation and had hypotension with mild shortness of breath.

\section{A repeat computed tomography} angiography to evaluate for pulmonary embolism, EKG scan, echocardiography scan, and MRI scan of the brain were all essentially unchanged from the time of last discharge. The patient was started on prednisone, $40 \mathrm{mg}$, twice daily, and her symptoms began to improve. She was subsequently discharged on hospital day 2. Our patient followed up regularly with the rheumatologist in the outpatient clinic and underwent a slow taper of prednisone over 3 months. To date, 8 months since her initial presentation, the patient has not shown any clinical or laboratory signs of disease re-emergence.

\section{Conclusion}

Although the pathophysiology of MIS-C and MIS-A remains unclear, our case report represents one complex presentation of this rare syndrome. Given the multiorgan involvement of this disease, we anticipate that variations in the presentation along with possible negative COVID-19 testing may make diagnosis of MIS-C/MIS-A difficult. Consequently, clinicians will have to maintain a high index of suspicion, as well as consider glucocorticoids early in the diagnosis and treatment of patients who present similarly to ours to prevent irreversible organ damage or death.

\section{References}

1. Multisystem inflammatory syndrome. Centers for Disease Control and Prevention. Reviewed June 25, 2021. Accessed August 12, 2021. https://www.cdc.gov/mis-c/hcp/

2. Morris SB, Schwartz NG, Patel P, et al. Case series of multisystem inflammatory syndrome in adults associated with SARSCoV-2 infection - United Kingdom and United States, March-August 2020. MMWR Morb Mortal Wkly Rep. 2020;69(40):14501456. https://doi.org/10.15585/mmwr. mm6940e1

3. Zhu N, Zhang D, Wang W, et al. A novel coronavirus from patients with pneumonia in China, 2019. N Engl J Med. 2020;382(8):727-733. https://doi. org/10.1056/nejmoa2001017

4. Guan WJ, Ni ZY, Hu Y, et al. Clinical characteristics of coronavirus disease 2019 in China. N Engl J Med. 2020;382(18):1708-1720. https://doi.org/10.1056/nejmoa2002032 
5. Huang C, Wang Y, Li X, et al. Clinical features of patients infected with 2019 novel coronavirus in Wuhan, China. Lancet. 2020;395(10223):497-506. https://doi. org/10.1016/s0140-6736(20)30183-5

6. Yang J, Zheng Y, Gou X, et al. Prevalence of comorbidities and its effects in patients infected with SARS-CoV-2: a systematic review and meta-analysis. Int J Infect Dis. 2020;94:91-95. https://doi.org/10.1016/j. ijid.2020.03.017

7. Khan IH, Zahra SA, Zaim S, Harky A. At the heart of COVID-19. J Card Surg. 2020;35(6):1287-1294. https://doi. org/10.1111/jocs.14596

8. Mao $L$, Jin $H$, Wang $M$, et al. Neurologic manifestations of hospitalized patients with coronavirus disease 2019 in Wuhan, China. JAMA Neurol, 2020;77(6):683-690. https:// doi.org/10.1001/jamaneurol.2020.1127

9. Naicker S, Yang CW, Hwang SJ, Liu BC, Chen JH, Jha V. The novel coronavirus 2019 epidemic and kidneys. Kidney Int. 2020;97(5):824-828. https://doi. org/10.1016/j.kint.2020.03.001

10. Wong SH, Lui RN, Sung JJ. Covid-19 and the digestive system. J Gastroenterol Hepatol. 2020;35(5):744-748. https://doi.org/10.1111/ jgh.15047

11. Combes AJ, Courau T, Kuhn NF, et al. Global absence and targeting of protective immune states in severe COVID-19 [Preprint]. Res Sq. Published online October 28, 2020. https://doi.org/10.21203/rs.3.rs-97042/v1

12. Park A, Iwasaki A. Type I and Type III interferons - induction, signaling, evasion, and application to combat COVID-19. Cell Host Microbe. 2020;27(6):870-878. https://doi. org/10.1016/j.chom.2020.05.008

13. Blanco-Melo D, Nilsson-Payant BE, Liu WC, et al. Imbalanced host response to SARSCoV-2 drives development of COVID-19. Cell. 2020;181(5):1036-1045.e9. https://doi. org/10.1016/j.cell.2020.04.026

14. Verdoni L, Mazza A, Gervasoni A, et al. An outbreak of severe Kawasaki-like disease at the Italian epicentre of the SARS-CoV-2 epidemic: an observational cohort study. Lancet. 2020;395(10239):1771-1778. https:// doi.org/10.1016/s0140-6736(20)31103-x

15. Chiotos K, Bassiri $\mathrm{H}$, Behrens EM, et al. Multisystem inflammatory syndrome in chil- dren during the coronavirus 2019 pandemic: a case series. J Pediatric Infect Dis Soc. 2020;9(3):393-398. https://doi.org/10.1093/ jpids/piaa069

16. Feldstein LR, Rose EB, Horwitz SM, et al. Multisystem inflammatory syndrome in U.S. children and adolescents. $N$ Engl J Med. 2020;383(4):334-346. https://doi. org/10.1056/nejmoa2021680

17. Ramcharan $\mathrm{T}$, Nolan $\mathrm{O}$, Lai CY, et al. Paediatric inflammatory multisystem syndrome: temporally associated with SARS-CoV-2 (PIMS-TS): cardiac features, management and short-term outcomes at a UK tertiary paediatric hospital. Pediatr Cardiol. 2020;41(7):1391-1401. https://doi. org/10.1007/s00246-020-02391-2

18. Abdel-Mannan $O$, Eyre $M$, Löbel $U$, et al. Neurologic and radiographic findings associated with COVID-19 infection in children. JAMA Neurol. 2020;77(11):14401445. https://doi.org/10.1001/jamaneurol.2020.2687

19. Schupper AJ, Yaeger KA, Morgenstern PF. Neurological manifestations of pediatric multi-system inflammatory syndrome potentially associated with COVID-19. Childs Nerv Syst. 2020;36(8):1579-1580. https:// doi.org/10.1007/s00381-020-04755-8

20. Kremer S, Lersy F, de Sèze J, et al. Brain MRI findings in severe COVID-19: a retrospective observational study. Radiology. 2020;297(2):E242-E251. https://doi. org/10.1148/radiol.2020202222

21. Puntmann VO, Carerj ML, Wieters I, et al. Outcomes of cardiovascular magnetic resonance imaging in patients recently recovered from coronavirus disease 2019 (COVID-19). JAMA Cardiol. 2020;5(11):12651273. https://doi.org/10.1001/jamacardio.2020.3557

22. Sanghvi SK, Schwarzman LS, Nazir NT. Cardiac MRI and myocardial injury in COVID-19: diagnosis, risk stratification and prognosis. Diagnostics (Basel). 2021;11(1):130. https://doi.org/10.3390/diagnostics 11010130 